BULLETIN OF THE

AMERICAN MATHEMATICAL SOCIETY

Volume 77, Number 4, July 1971

\title{
SEMIAPOSYNDETIC NONSEPARATING PLANE CONTINUA ARE ARCWISE CONNECTED
}

\author{
BY CHARLES L. HAGOPIAN ${ }^{1}$
}

Communicated by Steve Armentrout, August 3, 1970

It is known that if $H$ is an aposyndetic nonseparating plane continuum, then $H$ is locally connected. This follows from a result of Jones' [2, Theorem 10] that if $p$ is a point of a plane continuum $H$ and $H$ is aposyndetic at $p$, then the union of $H$ and all but finitely many of its complementary domains is connected im kleinen at $p .{ }^{2}$ As a corollary of these results, each aposyndetic nonseparating plane continuum is arcwise connected. Closely related to the notion of an aposyndetic continuum is that of a semiaposyndetic continuum, studied in [1]. A continuum $M$ is semiaposyndetic if for each pair of distinct points $x$ and $y$ of $M$, there exists a subcontinuum $F$ of $M$ such that the sets $M-F$ and the interior of $F$ relative to $M$ each contain a point of $\{x, y\}$. Note that a nonseparating semiaposyndetic plane continuum may fail to be locally connected. The main theorem of this paper is that each semiaposyndetic nonseparating plane continuum is arcwise connected. A complete proof of this result will appear elsewhere. For definitions of unfamiliar terms and phrases see [4].

Throughout this paper $S$ is the plane and $d$ is the Euclidean metric for $S$.

Definition. Let $E$ be an arc-segment (open arc) in $S$ with endpoints $a$ and $b, D$ be a disk in a continuum $M$ in $S$, and $\epsilon$ be a positive real number. The arc-segment $E$ is said to be $\epsilon$-spanned by $D$ in $M$ if $\{a, b\}$ is a subset of $D$ and for each point $x$ in a bounded complementary domain of $D \cup E$, either $d(x, E)<\epsilon$ or $x$ belongs to $M$.

Definition. A point $y$ of a continuum $M$ cuts $x$ from $z$ in $M$ if $x, y$

AMS 1969 subject classifications. Primary 5475, 5455; Secondary 5422.

Key words and phrases. Nonseparating plane continua, aposyndesis, semiaposyndesis, arc-wise connectedness.

1 The author gratefully acknowledges the help and encouragement of Professors E. E. Grace and F. B. Jones.

${ }^{2}$ A continuum $H$ is said to be aposyndetic at a point $p$ of $H$ with respect to a point $q$ of $H-\{p\}$ if there exist an open set $U$ and a continuum $L$ in $H$ such that $p \in U \subset L$ $C H-\{q\}$. A continuum $H$ is said to be aposyndetic at a point $p$ if for each point $q$ of $H-\{p\}, H$ is aposyndetic at $p$ with respect to $q$. If $H$ is aposyndetic at each of its points, then $H$ is said to be aposyndetic (Jones). 
and $z$ are distinct points of $M$ and $y$ belongs to each subcontinuum of $M$ which contains $\{x, z\}$.

The following lemmas are necessary preliminaries.

LEMMA 1. If an arc-segment $E$ in $S$ of diameter less than $\epsilon$ with endpoints $a$ and $b$ is $\epsilon$-spanned by a disk $D$ in $M$ (a continuum in $S)$, then there exists an arc-segment $M(E)$ in $M$ with endpoints $a$ and $b$ such that for each point $x$ of $M(E), d(x, E) \leqq 2 \epsilon$.

LEMмa 2. If $M$ is a semiaposyndetic metric continuum and $x, y$ and $z$ are points of $M$ such that $y$ cuts $x$ from $z$ in $M$, then $z$ does not cut $x$ from $y$ in $M$.

Theorem. If $M$ is a semiaposyndetic continuum in $S$ which does not separate $S$, then $M$ is arcwise connected.

Proof. (Sketch). Let $p$ and $q$ be distinct points of $M$. According to Jones' cyclic connectivity theorem [3], if no point cuts $p$ from $q$ in $M$, then $p$ and $q$ belong to a simple closed curve in $M$ and are therefore the extremities of an arc lying in $M$. Suppose there exists a point which cuts $p$ from $q$ in $M$. Let $K$ be the closed subset of $M$ consisting of $p, q$ and all points $x$ such that $x$ cuts $p$ from $q$ in $M$. Define the binary relation $R$ on $K$ as follows. For distinct points $x$ and $y$ of $K$, $x R y$ if $x$ cuts $p$ from $y$ in $M$ or $x=p$. Using Lemma 2, one can prove that $R$ is a natural ordering of $K$ as defined by G. T. Whyburn [5, p. 41]. Hence there exists an arc $A$ not necessarily in $S$ containing $K$ such that $p$ and $q$ are endpoints of $A$ and $R$ is the order induced on $K$ from $A[5$, Theorem 6.4, p. 56].

Let $E$ be a component of $A-K$ with endpoints $a$ and $b$. Assume without loss of generality that either $a$ cuts $p$ from $b$ in $M$ or $a=p$. Suppose there exists a point $x$ such that $x$ cuts $a$ from $b$ in $M$. One can prove that the point $x$ belongs to $K, a R x$ and $x R b$. Hence $x$ must belong to $E$. This contradicts the assumption that $E$ is a subset of $A-K$. Therefore no point cuts $a$ from $b$ in $M$. Let $C$ denote the set of components of $A-K$. It follows from Jones' cyclic connectivity theorem that for each element $E$ of $C$, there exists a simple closed curve $J(E)$ in $M$ which contains the endpoints of $E$. Since $M$ does not separate $S$, there exists a disk $N(E)$ in $M$ such that the endpoints of $E$ are in $N(E)$. Note that if $C$ is finite, one can easily define an arc in $M$ with endpoints $p$ and $q$.

Assume that $C$ is infinite. For each element $E$ of $C$ define $E^{*}$ to be the straight line segment in $S$ which has the endpoints of $E$ as endpoints. Since $M$ is semiaposyndetic, for each positive real number $\epsilon$, the set consisting of all elements $E$ of $C$ such that $E^{*}$ is not $\epsilon$-spanned 
by a disk in $M$ is finite. For each positive integer $n$, let $C_{n}$ be the finite set consisting of all elements $E$ of $C$ such that either the diameter of $E^{*}$ is greater than or equal to $1 / 2 n$, or $E^{*}$ is not $1 / 2 n$-spanned by a disk in $M$. Let $H_{1}=C_{1}$, and, for $n=2,3,4, \cdots$, let $H_{n}=C_{n}$ $-C_{n-1}$. For each element $E$ of $C$, define the arc-segment $M(E)$ as follows. Assume that $a$ and $b$ are the endpoints of $E$. There exists an integer $n$ such that $E$ belongs to $H_{n}$. If $n=1$, define $M(E)$ to be an arc-segment in $N(E)$ with endpoints $a$ and $b$. According to Lemma 1, if $n>1$, there exists an arc-segment $M(E)$ in $M$ with endpoints $a$ and $b$ such that for each point $x$ of $M(E), d\left(x, E^{*}\right) \leqq 1 /(n-1)$. One can prove that for each element $X$ of $C,\left(K \cup \bigcup_{E \in C-\{X\}} M(E)\right) \cap M(X)$ $=\varnothing$. For each element $E$ of $C$, let $f_{E}$ be a homeomorphism from $E$ onto $M(E)$. Define the function $f$ from $A$ to $K \cup \cup_{E \in C} M(E)$ as follows. For each point $x$ of $K$, define $f(x)=x$. If $x$ is a point of $A-K$, define $f(x)=f_{E}(x)(x \in E)$. The function $f$ is a homeomorphism. Hence $K \cup \bigcup_{E \in C} M(E)$ is an arc in $M$ from $p$ to $q$. It follows that $M$ is arcwise connected.

\section{BIBLIOGRAPHY}

1. C. L. Hagopian, Arcwise connectedness of semiaposyndetic plane continua, Trans. Amer. Math. Soc. 158 (1971) (to appear).

2. F. B. Jones, Aposyndetic continua and certain boundary problems, Amer. J. Math. 63 (1941), 545-553. MR 3, 59.

3. - The cyclic connectivity of plane continua, Pacific J. Math. 11 (1961), 1013-1016. MR 25 \#2583.

4. R. L. Moore, Foundations of point set theory, rev. ed., Amer. Math. Soc. Colloq. Publ., vol. 13, Amer. Math. Soc., Providence, R. I., 1962.

5. G. T. Whyburn, Analytic topology, rev. ed., Amer. Math. Soc. Colloq Publ., vol. 28, Amer. Math. Soc., Providence, R. I., 1963. MR 32 \#25.

Sacramento State College, Sacramento, California 95819 